\title{
Teaching trainees neuraxial anaesthesia. What is the evidence?
}

\author{
James Garwood*1,2 and Brian Wilkinson ${ }^{3}$ \\ ${ }^{1}$ University of Exeter Medical School (UEMS), St. Luke's Campus, United Kingdom \\ ${ }^{2}$ Department of Anaesthetics, Royal Devon and Exeter NHS Foundation Trust, United Kingdom \\ ${ }^{3}$ Improvement Academy, Bradford Institute for Health Research, Bradford Royal Infirmary, United Kingdom
}

Submission: February 18, 2020; Published: February 28, 2020

*Corresponding author: James Garwood, Department of Anaesthetics, Royal Devon and Exeter NHS Foundation Trust, Barrack Rd, Exeter, EX2 5DW, United Kingdom.

\begin{abstract}
Summary
Central neuraxial blockade (CNB) describes a range of procedures that are used to provide perioperative anaesthesia and/or analgesia for patients undergoing a wide variety of surgical procedures. It represents a significant proportion of workload for both anaesthetists and those in training. The most effective method(s) for teaching and training novice anaesthetists to perform CNB remains unclear. This paper presents a systematic review of the literature and recommendations for educational techniques in clinical practice. A systematic review of the literature was undertaken. Medline, PubMed, ERIC, Cochrane library, PsycInfo, and HMIC databases were searched from inception to June 2018 for studies involving CNB skills teaching. Searches were limited to the English language and full text availability. Studies were analysed and quality scored using the MERSQI scale [1]. Outcomes for each intervention were also plotted against a modified Kirkpatrick's model. 14 papers ( $\mathrm{n}=14$ ) were included in the review. The mean MERSQI score was 12.43 (range 8.5-16). 9 of the studies had a MERSQI score above 12. The studies reported a range of educational and clinical outcomes; the modal outcome was improved knowledge and/or skills (2b). Interventions identified were the use of Ultrasound ( 2 studies), Educational multimedia (4 studies), Simulation (8 studies), Feedback delivery (1 study), Mental imagery (1 study), and Learning curves ( 1 study). The use of ultrasound imaging and simulated clinical practice demonstrated the most significant effects on trainee knowledge and skill; however, studies were generally small, and as both methods and outcomes showed marked heterogeneity, large scale, high quality studies would be beneficial to this important aspect of anaesthetic practice.

Abbreviations: ASME: Association for the Study of Medical Education; BEME: Best Evidence Medical and Health Professional Collaboration; CCT: Completion of Certificate of Training; CNB: Central Neuraxial Blockade; CSE: Combined Spinal-Epidural; CUSUM: Cumulative Sum Control; EM: Educational Multimedia; EMS: Emergency Medical Services; ERIC: Education Resources Information Centre; GMC: General Medical Council; HMIC: Healthcare Management Information Consortium; MERSQI: Medical Education Research Study Quality Instrument; NOS: NewcastleOttawa scale; PDPH: Post Dural Puncture Headache; PICO: Participants, Intervention, Comparison, Outcome; PRISMA: Preferred reporting items for systematic reviews and meta-analyses; RCoA: Royal College of Anaesthetists; RCT: Randomised Controlled Trial; REC: Research Ethics Committee; UK: United Kingdom; USA: United States of America
\end{abstract}

\section{Introduction}

Central neuraxial blockade (CNB) is a type of regional anaesthesia used by anaesthetists and anaesthesiologists to provide both anaesthesia and analgesia for patients, for a wide range of procedures, with the added benefit of a reduction in morbidity after major surgery [2-4]. Over 700,000 (Epidural, Spinal, Combined Spinal-Epidural (CSE) and Caudal) neuraxial anaesthetic procedures are performed annually in the United Kingdom [5]. Epidural catheter insertion is the most common central neuraxial procedure performed by anaesthetic trainees in the United Kingdom. This is most commonly for the provision of intrapartum analgesia during childbirth [6]. All doctors in anaesthetic training must be able to demonstrate competence in

performing CNB as part of their core and intermediate training certificates required for award of certificate of completion of specialist training (CCT) by the Royal College of Anaesthetists (RCoA) and entry onto the General Medical Council (GMC) Specialist Register $[7,8]$. How we introduce and train our novice and core trainees to perform CNB has been a topic of discussion amongst anaesthetic educators for some time [9]. Teaching trainees how to perform CNB safely has traditionally followed a 'see one, do one' philosophy [10], with often more emphasis placed on novice trainees learning by observing competent practitioners performing CNB insertion; and subsequent direct clinical 'practice' with progressively reducing levels of supervision. Although the 
risks of CNB are low, around 1 in 23500 recipients [5] will develop a permanent neurological injury. The effect of these complications can be life changing. Furthermore, inadvertent dural puncture has an overall incidence of around 1:100 epidural insertions but occurs more frequently for inexperienced operators. The likely subsequent post-dural puncture headache (PDPH) seen with large Tuohy needles can be debilitating and impact upon a new mother's ability to care for her baby. Whilst the course of PDPH is generally self-limiting, within a seven-to-ten-day period, PDPH can increase hospital length of stay, cost of treatment and require further procedures such as epidural blood patching [5]. It is our responsibility, as educators, to ensure we can minimise these risks to patients as much as possible, whilst ensuring maximum effect of our teaching and training opportunities. Learning procedural skills requires three stages of cognition, integration, and automation [11]. How this is best achieved has been the subject of much debate and research [10-13]. Research in the area of education relating to practical skills has so far been pioneered by our surgical colleagues [14]. Simulation may have a significant beneficial role in the development of some procedural skills $[14,15]$ and has the benefit of being deliverable away from direct patient care, as it can also be difficult to provide protected training time within theatre lists due to system pressure to achieve maximum efficiency. Simulation also reduces patient exposure to risk during the time when the learner is at their most inexperienced, with novel equipment and techniques. As well as simulation, educational courses, application of different models of learning, and methods of assessing and determining procedural competence have all been researched within the literature $[10,14]$. Despite the broad coverage of research; there is very limited evidence on what methods have been proven to be effective in teaching procedural skills to anaesthetic trainees. With CNB forming a significant part of obstetric, regional, vascular and orthopaedic anaesthesia, it is important for educators to be up to date with the best evidence for teaching these techniques. As the shift in post-graduate training moves towards competency-based training, assessment of individual skills and procedures becomes even more prominent. We still need to find an effective way of teaching CNB skills to our trainees, to enhance their learning opportunities in the best way possible.

\section{Research question}

'The research question to be addressed was 'Which educational intervention is most effective in teaching trainees central neuraxial anaesthesia?'

\section{Methods}

This systematic review was planned, conducted, and reported according to 'preferred reporting items for systematic reviews and meta-analyses' (PRISMA) standards [16]. A research question was developed using a PICO (Participants, Intervention, Comparison, Outcome) method. A search protocol was written to guide search methods and explicitly define inclusion and exclusion criteria, and to guide how the quality of results was to be interpreted and evaluated.

\section{Eligibility criteria}

To ensure the widest possible search, the eligibility of studies to be included in the search were: English language and any patient (as all age groups may potentially receive neuraxial anaesthesia). The trainee group could be either undergraduate or postgraduate, and related to any of medicine, nursing, and all other allied health professionals (paramedic/EMS, physician associates, physiotherapists etc.). The studies needed to involve to human subjects/patient groups or be the intended end user group to the intervention. Studies needed to be based upon either teaching or educational methods in reference to neuraxial anaesthesia or regional anaesthesia. It was decided to include the more generic term of regional anaesthesia within the eligibility as there is a degree of crossover on how anaesthetists may define or classify different types of neuraxial anaesthesia [17]. Additionally, there is a great deal of commonality in the skills required to perform neuraxial anaesthesia, lumbar puncture and peripheral nerve blockade, therefore studies assessing these procedures were also deemed eligible for inclusion [18].

\section{Information sources}

\section{Search}

Medline, PubMed, ERIC, Cochrane library, PsycInfo, and HMIC databases were used as primary information sources. The databases were searched from their dates of inception to June 2018. The final search was undertaken on $6^{\text {th }}$ June 2018. A full electronic search strategy of one of the databases can be seen in Appendix 1, but in summary the search terms were linked to the population (anaesthetists), intervention (training or teaching/ education), and procedure (neuraxial anaesthesia). The terms were mapped to 'Medical Subject Headings' (MeSH) heading where possible, and consideration of variations in spelling, e.g. anaesthetist in the UK, vs anaesthesiologist in the USA were also taken into account, as were geographical variations in terminology for trainees e.g. residents or interns.

\section{Study selection}

The principal author (JG) reviewed abstracts from all papers returned in the database search. Reviews, opinion articles, letters, conference abstracts, surveys, and guidelines were all excluded. The remaining results from the search were then retrieved in full, and the inclusion/exclusion process was repeated after full analysis of each paper. If the author was unable to choose if a study should be included, an independent colleague was consulted for advice, with a consensus decision made.

\section{Data collection process}

Data from the selected group of studies was then extracted using a proforma (Appendix 2). Data on study type, aims, trainee demographics, intervention, bias, and outcomes were recorded. 
Studies were evaluated using two methods. Initially the quality of studies was scored using the Medical Education Research Study Quality Instrument (MERSQI) [19], then the achieved outcomes of the study were also graded against a modified Kirkpatrick scale [20]. Data extracted was summarised into tables and quantitative analysis of MERSQI scores was performed. Bias of trials was assessed using the Cochrane Collaboration tool [21,22].

\section{Results}

Database search yielded a total of 115 abstracts which were assessed against inclusion/exclusion criteria. After initial screening, 52 studies were excluded from search, and 68 studies were retrieved for full analysis. 54 studies were subsequently excluded after full content review. The flowchart of the search, with reasons for exclusion can be seen in Figure 1. A summary of data extracted from included studies is seen in Appendix 3. A complete breakdown of each paper retrieved with reasons for exclusion can be seen in Appendix 4. Of the $14(n=14)$ studies which were included, eight were randomised controlled trials and six were single cohort studies. Students were nearly unanimously postgraduate trainees, with only one of the fourteen studies reporting an educational intervention for an undergraduate group. In total there were 372 trainees in all studies, of whom 307 (83\%) were postgraduates and $65(17 \%)$ were undergraduates. Procedure breakdown for anaesthesia type was epidural (7 studies), spinal (4 studies), and peripheral nerve blocks (3 studies). The educational interventions assessed in each study described six main methods. Two studies used a combination of interventions [23,24]. The use of simulation or a simulator model was the most frequently described educational tool and was seen in $8(57 \%)$ studies. The remaining methods were the use of ultrasound ( 2 studies), a professionally created educational training video ( 3 studies), differing methods of feedback (1 study), mental imagery (1 study), and development of learning curves (1 study). The most frequently reported study outcomes were improved knowledge and skills (reported/assessed by whom?) by 10 studies (71\%). Other reported outcomes were improved perception of knowledge ( 1 study), behavioural change (1 study), organisational change ( 2 studies), and one study reported changes to trainee learning (or improvement for patients). A full summary of demographics from all studies reviewed is presented in Table 1.

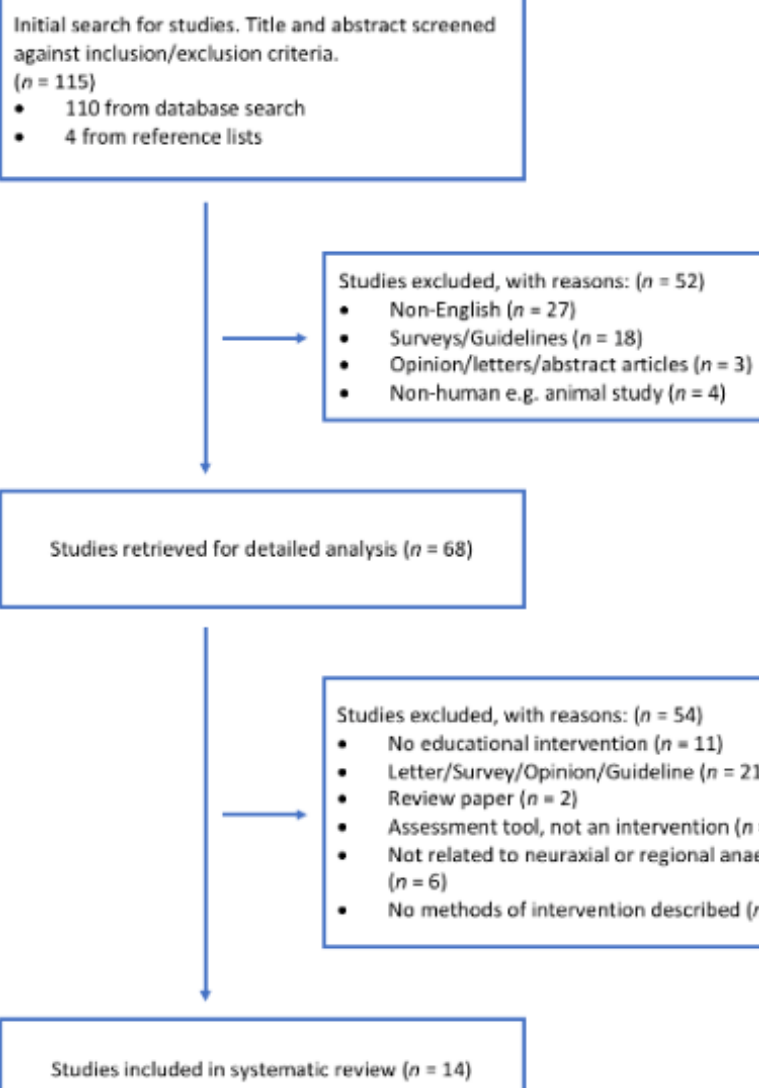

Studies included in systematic review $(n=14)$

Figure 1: Search Flow Chart 
Table 1: Demographics of studies in systematic review.

\begin{tabular}{|c|c|c|c|}
\hline Study Domain & \multicolumn{2}{|c|}{ Variable } & No. of studies (No. of trainees) \\
\hline \multicolumn{3}{|c|}{ All Studies } & $14(372)$ \\
\hline \multirow{2}{*}{ Design } & \multicolumn{2}{|c|}{ Randomised Controlled Trial (RCT) } & $8(223)$ \\
\hline & \multicolumn{2}{|c|}{ Single Cohort } & $6(149)$ \\
\hline \multirow{6}{*}{ Intervention ${ }^{\text {gh }}$} & \multicolumn{2}{|c|}{ Ultrasound } & $2(15)$ \\
\hline & \multicolumn{2}{|c|}{ Educational multimedia (EM) } & $4(127)$ \\
\hline & \multicolumn{2}{|c|}{ Simulation/Simulator } & $8(226)$ \\
\hline & \multicolumn{2}{|c|}{ Method of feedback delivery } & $1(65)$ \\
\hline & \multicolumn{2}{|c|}{ Mental imagery } & $1(20)$ \\
\hline & \multicolumn{2}{|c|}{ Learning curve } & $1(13)$ \\
\hline \multirow{2}{*}{ Trainee group } & \multicolumn{2}{|c|}{ Postgraduate } & $13(307)$ \\
\hline & \multicolumn{2}{|c|}{ Undergraduate } & $1(65)$ \\
\hline \multirow{3}{*}{ Procedure } & \multicolumn{2}{|c|}{ Epidural } & 7 (119) \\
\hline & \multicolumn{2}{|c|}{ Spinal } & $4(48)$ \\
\hline & \multicolumn{2}{|c|}{ Peripheral Nerve Block } & $3(71)$ \\
\hline \multirow{6}{*}{ Outcomes ${ }^{\text {aथ }}$} & Participation & Level 1 & - \\
\hline & Attitudes or perception & Level 2a & $1(50)$ \\
\hline & Knowledge and Skills & Level 2b & $10(293)$ \\
\hline & Behavioural changes & Level 3 & $1(40)$ \\
\hline & Organisational changes & Level 4a & $2(29)$ \\
\hline & Trainee learning changes & Level 4b & $1(10)$ \\
\hline Quality $^{\mathrm{b}}$ & \multicolumn{2}{|c|}{ MERSQI Score $\geq 12$} & $9(234)$ \\
\hline
\end{tabular}

aHierarchy of increasing importance. Adapted from BEME Guide No. 8 [19].

'Studies $\geq 12$ on MERSQI score shown to be of higher quality by Reed et al (2007) [18].

${ }^{\mathscr{H}}$ Collective number may exceed total study's $(\mathrm{n})$ if individual study had more than one stated primary outcome or intervention.

\section{Quality of studies}

A summary of MERSQI domain breakdown is presented in Table 2. The mean MERSQI score was 12.43 (range 8.5 - 16) with a possible score from 5 (minimum) - 18 (maximum). The median score was 12.5 . Nine out of the fourteen studies had MERSQI scores greater than or equal to 12 [23,25-32]. The study design comprised two main categories. Half of the studies were randomised controlled trials (7 studies) and the remainder were single cohort studies, which could be further subdivided, into post interventional assessment of a single cohort (4 studies), or a single cohort with pre- and post-intervention assessments (3 studies). 13 of the studies only reported results from a single institution, with one study giving results from 3 institutions [33]. Assessment data was assessed by authors for all studies. Validity varied across the studies, with the majority of studies reporting internal structure, content, and relationships to other variables of their evaluation instrument. Generally higher scoring studies had more clearly defined and validated evaluation methods [23,2532]. Analysis was generally appropriate for study design, except one study which gave no quantitative analysis of the data obtained [34]. From a complexity viewpoint, 7 studies gave only descriptive analysis [23,27,30,33-36], whereas the other 7 studies gave a more complex and in-depth analysis of their data [24-26,28,29,31,32]. MERSQI scores were generally closely matched to the outcomes which had been drawn out of the studies as a separate data-point during data collection. There was a discrepancy between some studies, which were scored as $4 \mathrm{a}$ and $4 \mathrm{~b}$ on the outcomes analysis (Table 1), and the scores awarded by the MERSQI outcomes, which were not awarded a higher score due to not matching the score descriptor.

Table 2: MERSQI analysis of studies by domain.

\begin{tabular}{|c|c|c|c|c|c|}
\hline Domain & MERSQI Item & Score & Max. Score & Frequency & $\%$ of studies \\
\hline \multirow{4}{*}{$\begin{array}{l}\text { Study De- } \\
\text { sign }\end{array}$} & Single group cross-sectional or single post-test only & 1 & \multirow{4}{*}{3} & 4 & $28.6 \%$ \\
\hline & Single group pre-test and post-test & 1.5 & & 3 & $21.4 \%$ \\
\hline & Non-randomised, 2 groups & 2 & & - & \\
\hline & Randomised controlled trial (RCT) & 3 & & 7 & $50.0 \%$ \\
\hline
\end{tabular}


Journal of Anesthesia \& Intensive Care Medicine

\begin{tabular}{|c|c|c|c|c|c|}
\hline \multirow{9}{*}{ Sampling } & No. of Institutions & & \multirow{9}{*}{3} & & \multirow[b]{2}{*}{$92.9 \%$} \\
\hline & 1 & 0.5 & & 13 & \\
\hline & 2 & 1 & & - & \\
\hline & 3 & 1.5 & & 1 & $7.1 \%$ \\
\hline & Response rate (\%): & & & & \\
\hline & not applicable & 0 & & 1 & $7.1 \%$ \\
\hline & $<50 \%$ or not reported & 0.5 & & - & \\
\hline & $50-74 \%$ & 1 & & - & \\
\hline & $>/=75$ & 1.5 & & 13 & $92.9 \%$ \\
\hline \multirow{2}{*}{ Type of data } & Assessment by participants & 1 & \multirow{2}{*}{3} & - & \\
\hline & Objective measurement & 3 & & 14 & $100.0 \%$ \\
\hline \multirow{12}{*}{$\begin{array}{l}\text { Validity of } \\
\text { evaluation } \\
\text { instrument }\end{array}$} & Internal structure: & & \multirow{12}{*}{3} & & \\
\hline & Not applicable & - & & - & \\
\hline & Not reported & 0 & & 4 & $28.6 \%$ \\
\hline & Reported & 1 & & 10 & $71.4 \%$ \\
\hline & Content: & & & & \\
\hline & Not applicable & - & & - & \\
\hline & Not reported & 0 & & 5 & $35.7 \%$ \\
\hline & Reported & 1 & & 9 & $64.3 \%$ \\
\hline & Relationships to other variables: & & & & \\
\hline & Not applicable & - & & 1 & $7.1 \%$ \\
\hline & Not reported & 0 & & 7 & $50.0 \%$ \\
\hline & Reported & 1 & & 6 & $42.9 \%$ \\
\hline \multirow{6}{*}{$\begin{array}{l}\text { Data ana- } \\
\text { lysis }\end{array}$} & Appropriate of analysis: & & \multirow{6}{*}{3} & & \\
\hline & Inappropriate for study design & 0 & & 1 & $7.1 \%$ \\
\hline & Appropriate for study design & 1 & & 13 & $92.9 \%$ \\
\hline & Complexity of analysis: & & & & \\
\hline & Descriptive & 1 & & 7 & $50.0 \%$ \\
\hline & Beyond descriptive & 2 & & 7 & $50.0 \%$ \\
\hline \multirow{4}{*}{ Outcomes } & Satisfaction, attitudes, perceptions, opinions, general facts & 1 & \multirow{4}{*}{3} & - & \\
\hline & Knowledge, Skills & 1.5 & & 9 & $64.3 \%$ \\
\hline & Behaviours & 2 & & 5 & $35.7 \%$ \\
\hline & Patient/healthcare outcome & 3 & & - & \\
\hline Total & & & 18 & & \\
\hline
\end{tabular}

\section{Interventions}

From results summary table in Appendix 2, there were 6 main interventions which were described in the studies.

\section{Simulation}

Simulation, a simulator device, or a phantom anatomy (e.g. spinal-epidural manikin [37]) was the main educational intervention for 8 of the 14 studies [24,26,28,30,33-35]. 226 trainees were included in the studies examining simulation. Five studies were RCTs, with the other three being single cohort with pre-and post-intervention testing. The mean MERSQI score for this intervention group was 12.0 (Range 8.5-16). Simulation when used as an educational tool to teach neuraxial anaesthesia compared against didactic teaching was shown to have a statistically significant improvement in successful procedure completion [28]. Two studies did not demonstrate an advantage for high-fidelity over low-fidelity simulation [26,28]. One study showed no difference in the rate of inadvertent dural puncture during epidural insertion between those who had trained on a simulator against those who had received standard teaching. However, trainees reported that simulation training reduced their perception of stress during subsequent real clinical procedures [2]. Use of a simulator prior to performing epidural injection, was associated with a significant reduction in time required to complete the procedure [35]. The use of simulators was associated with a small increase in the ability to identify anatomy in one study, however this fell short of statistical significance [30]. When length of exposure to a simulator was compared to completion 
time, there was a directly proportional link [34]. A study of Emergency Medicine residents performing simulated femoral nerve block showed the subjects retained $85 \%$ of their knowledge after 3 months [33]. This was a single, small cohort however, the generalisability of the conclusions remain uncertain. In the studies bias was mitigated by randomisation, control groups, and the use of validated scoring check lists, and it is the authors' opinions that the reviewed studies achieved this to a reasonable degree.

\section{Educational multimedia (EM)}

Four out of fourteen studies used educational video, multimedia, or virtual reality as a single or part of a bundle of educational interventions [24,27,30,36]. 27 participants were included in Educational Multimedia studies, which had a mean MERSQI score of 11 (Range 9-13.5). A randomized control trial found no significant difference in (which outcome) comparing a simulator, instructional educational video, and didactic teaching. The professionally created multimedia video was as effective as simulation training, and there was a significant improvement in perceived knowledge [30]. Exposure of a single cohort of trainees to virtual reality animations was shown to improve surface marking knowledge and skill to perform a peripheral nerve block, but only the moderately experienced group gained a significant change in scores. The novice and expert group saw no significant change in their pre- and post-intervention scores [36]. (Friedman et al (2013) [26] demonstrated that the use of a professionally created educational video in a single cohort of anaesthesia trainees was associated with a statistically significant improvement in aseptic epidural insertion technique, as marked by blinded assessors. Kulcsár Z et al (2013) [23] found that the combination of virtual reality goggles and a simulator was not superior to conventional teaching for the performance of spinal anaesthesia [24].

\section{Ultrasound}

Only one study (Grau et al (2003) [24] yielded by the systematic review used ultrasound as an educational intervention. The RCT compared ultrasound-guided versus standard skin palpation as a tool prior to skin puncture during epidural insertion and compared two cohorts of novice trainees. The difference between the two groups' performances was stark such that the shape of the learning curve seen typically for epidural insertion was radically altered $(\mathrm{p}<0.001)$. In the skin palpation (control) group the authors observed a success rate of $60 \% \pm 16 \%$ after the first ten attempts followed by a learning curve where the subsequent 50 epidurals had an $84 \%$ success rate. Using ultrasound-guidance was associated with a much greater initial rate of success $(86 \% \pm 15 \%)$ and a higher success rate of $94 \%$ for the subsequent 50 insertions. The difference between the two groups remained significant at all points during the study. In total 600 epidurals were performed by 10 trainees for this study, making this one of the largest studies into education intervention for central neuraxial anaesthesia.

\section{Feedback Delivery}

Lean et al (2017) [31] compared timing of feedback delivery and its impact upon short-and long-term knowledge retention. 65 undergraduate trainees either received feedback from a supervisor during the performance of a spinal anaesthetic, or post procedure. The results showed that the group who had received feedback post-procedure were was superior with regards to successful trainee performance of spinal anaesthesia with reduced time to insertion.

\section{Mental Imagery}

A single RCT (Lim et al (2016)) [30] compared the use of a low-fidelity haptic simulator (banana) and the use of rehearsed mental imagery for epidural insertion by 20 postgraduate anaesthesia trainees. Both groups all showed equal levels of improvement over the 3 assessments using validated checklists and no significant differences were seen between the two techniques. The authors concluded that "structured didactics and continual mental imagery training may suffice to prepare novice learners before an attempt on human subjects."

\section{Learning curves}

Three studies used either the development of a learning curve with cumulative sum (CUSUM) statistical methods following analysis of an educational intervention [23,29], or an observational study to provide data such that a learning curve could be described for undertaking regional anaesthesia [34]. 27 postgraduate trainees were included in the studies. The CUSUM analysis used by two studies concluded that 57 epidural insertions were needed to become competent, (as defined by) whereas ultrasound scanning of a spine prior to inserting an epidural required 65-75 scans until competency was achieved $[23,29]$. This approximates with the learning curves described by Grau et al (2003) [24]

\section{Discussion}

This systematic review has identified 14 studies of educational strategies which either augment or replace traditional "see one, do one" teaching in neuraxial anaesthesia performance. The use of simulation was the most frequent intervention described in the literature and showed that overall it has a positive effect on learning. Simulation has the advantage of helping students gain procedural exposure whilst minimising the risk to patients at the start of the learning curve. Whilst several studies demonstrated global significant technical improvement and others noted a non-significant trend towards perceived knowledge, the improvements tended to represent the lower scale of hierarchy of outcomes. Simulation is an over-arching term that can be used to describe a range of modalities and techniques; however, this review raises questions regarding the optimal modality of simulation as an educational aid to neuraxial anaesthesia. Studies comparing high-tech fidelity simulators against simple low-tech 
simulators showed no difference in perceived competency or the ability to complete the procedure. Where a commercial epidural simulator may cost between $£ 8000$ - $£ 18000$ (GBP) [37], the cost of the fruit or vegetables that were typically used in the low-tech comparative arm was less than $£ 1$ each. Low-fidelity simulation was markedly less expensive and yielded similar results. In a time of austerity and budget cuts within medical education, we should be increasingly mindful of economics as educators $[26,38]$. These findings are also of interest to lesser economically developed healthcare settings. The simulation studies showed a wide range in quality, providing both the highest and lowest MERSQI scores in this review. If we compare the MERSQI scores to the outcome grading, there is an association between outcome level and higher MERSQI scores. This is partly explained by Kirkpatrick outcomes comprising part of the MERSQI scoring, meaning that they are not truly independent variables. Several forms of Educational Multimedia (EM) was represented in this review. Some of the higher MERSQI-rated studies used a professionally created video to standardise the teaching prior to testing trainees. However, this may have introduced bias as to the likely assessment criteria, i.e. the contents of the video rather than promoting a deeper understanding and application of procedural knowledge. Generally, the EM studies outcome were rated as $2 \mathrm{~b}$ 3 , demonstrating only improved knowledge, and some minor changes to behaviours. The MERSQI scores generally correlated with the Kirkpatrick outcomes. The single study (Grau et al, 2003) involving ultrasound as an intervention to improve epidural insertion was a significant paper. Although only a pilot study, it demonstrated that by teaching a trainee to ultrasound the spine prior to skin puncture, the success rate changed considerably, and altered the shape of the learning curve. The Kirkpatrick outcome was rated as $4 \mathrm{~b}$-the study has significant potential ramifications for novice anaesthetist training. With ultrasound being an increasingly available and core part of both education and clinical practice, strong consideration should be given to training epidural insertion with ultrasound from the beginning. If the results of the study were proven generalizable to widespread clinical practice, implementation of ultrasound-guided epidural insertion as a base technique would lead to significant increase in first pass rates, fewer accidental dural punctures, and a consequent reduction in the morbidity associated with neuraxial anaesthesia [39].

Timing of feedback delivery to trainees is an important consideration when teaching and coaching a practical skill. Evidence from this review demonstrates that feedback should be delivered after the encounter, away from the potentially judgemental ears of patients, colleagues, and other staff. Postprocedural feedback, as opposed to 'real-time' appears to improve skill retention. This may be explained by the concept of cognitive load. When learning a new skill, a trainee's 'bandwidth', i.e. the capacity to increase cognitive workload, is saturated, and the trainee is unable to assimilate feedback at that point. [40]. Mental imagery models serve as a means for preparing trainees with what could be considered 'pre-procedure feedback' and this review shows this may well improve performance. Taking the time to use mental imagery with trainees can be as effective as simulation in successful completion of a neuraxial anaesthesia. Walking through each practical step helps to lay the foundations for the cognitive phase of acquisition of a skill [41]. The majority of teaching and learning in anaesthesia can be contextualised as 'Situated Learning Theory' [42,43]. Skills and procedures are often discussed and/or demonstrated to a trainee prior to supervised undertaking, and this pre-procedural brief may have significant effects on trainee confidence, knowledge, and success rates. Learning curve development is an important concept for teachers within anaesthesia. Anaesthesia represents a set of skills that are seldom encountered during other clinical placements, and therefore the pathway from novice to mastery as defined by the curriculum is well-established. Understanding of learning curves allows trainers to anticipate the expected course of learning and identify deviance which may be amenable to either a prolongation or re-modelling of training. Learning curves are also important in workforce modelling as novice anaesthesia trainees will require direct supervision until they can achieve initial competencies, and the curves can help identify median trainees and outliers. Learning curves aid trainer's knowledge to be able to coach and provide feedback to a trainee, as to what to expect, and how will find their success rates change over time. This may provide reassurance when a novice trainee is at risk of feeling the competency appears insurmountable. Grau et al (2003) [24] demonstrated that the characteristics of a learning curve including its shape, steepness and plateau(s) can help provide a means of comparison between educational interventions with regards to an outcome. Kirkpatrick's model [44] provides the basis for the modified hierarchy described and adapted by the Best Evidence Medical and Health Professional Collaboration (BEME) for assessing study outcomes [20]. This scoring system generally is best placed for scoring simple educational interventions, such as the majority of those found in this systematic review. ${ }^{45}$ However Kirkpatrick's model may overestimate the outcome or give a false impression that an intervention is a significant one, when in fact the study may be a high risk of bias e.g. by having low numbers of participants . Conclusions should therefore be viewed cautiously [44]. One could argue that there may be a flaw with using the MERSQI scoring system, as the outcomes scoring domain within it has a smaller range and it may introduce an element of 'restrictive bias'. Expansion of the MERSQI outcomes domain may allow the tool to become a better discriminator for higher quality studies. Despite the review being focused upon a range of largely similar educational interventions, there was a wide range of MERSQI scores seen. The challenge lies in discriminating between poor educational interventions and poor methodology. The risk that a poor study design leads to an effective intervention being downgraded, and the converse risk also applies. The review demonstrates a significant lack of large RCTs, powered for definitive conclusions. Overall the BEME-graded outcomes (Table 1) were generally a $2 \mathrm{~b}$ score - demonstrating an increase 
in both procedural knowledge and skill. This is clearly a core component of anaesthetic training. As medical educators we should be aiming to devise both educational interventions and sound methodological practices that enhance trainee learning [45]. One way to improve anaesthetic trainee learning could be to adapt Kirkpatrick's model to become a reflective framework for planning an educational intervention by combining the model with an iterative interrogative technique, like those used in root cause analysis [46,47]. This adaptation of Kirkpatrick's model would allow the question to be posed as "How" can we improve our a) intervention, and b) research planning to reach the next level of outcomes? The model may also be considered as a reflective tool, asking "Why" at each level, did our intervention not reach this level, until a root cause can be established? The proposed model would allow us to ask both "how" can we reach the next level, or "why" didn't we reach this level? (Figure 2)

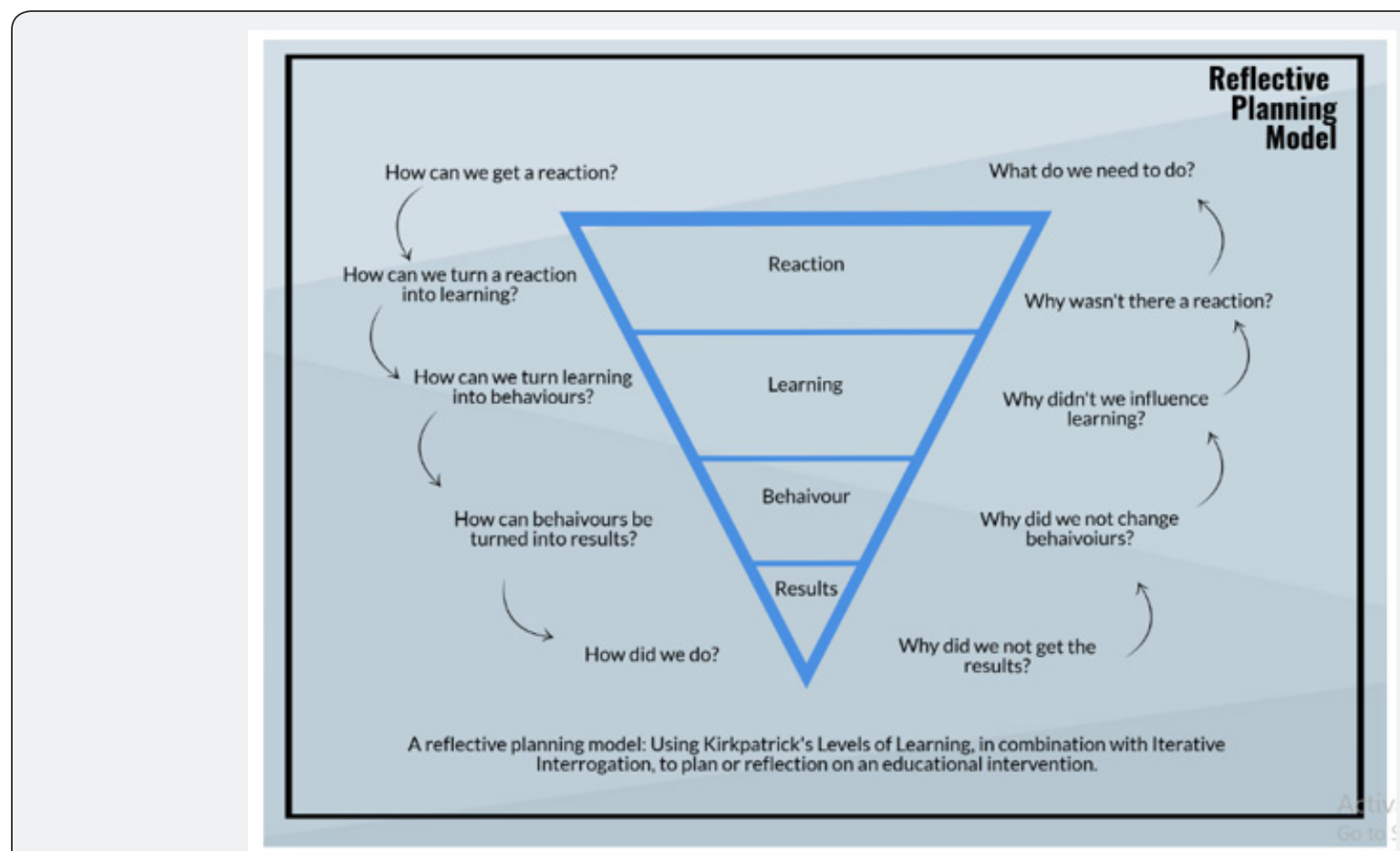

Figure 2: Proposed 'Reflective planning model'.

\section{Limitations}

This study is limited in several ways. The quality and quantity of the original research studies found in this study was quite low. This may indicate that the research question was too narrow, the inclusion criteria may have been too stringent, or that there may be just very limited evidence on teaching in this area of anaesthesia. All studies that were reported in the literature conferred a positive outcome. Studies yielding negative outcomes may not have been published and may skew the evidence artificially [48]. The MERSQI scoring system has been criticised in other literature for perhaps being limited in its use [45], and other scoring systems such as the Newcastle-Ottawa scale (NOS) [49] or the BEME global score [50], may have been a better comparison against quality of studies. As all studies in the review were assessed by a single author (JG), this will, despite the authors' best efforts, introduce bias into the study [22].

\section{Conclusions}

The overall evidence for the best method for teaching neuraxial anaesthesia to anaesthetic trainees remains limited. The lack of randomised control trials is particularly concerning. No single educational tool seemed to demonstrate marked superiority over another, and well-conducted studies comparing different techniques, or bundles of techniques, would greatly enrich the evidence base. This may represent the challenges of delivering RCTs (including the expense, time and ethical considerations). There needs to be development of higher quality research with increased numbers of trainees, to minimise the bias within smaller studies. We should standardise our research planning and development to allow for increase a more powerful evidence base-collaboration between institutions and considerations for an educational trial database could be considered. The development of the reflecting planning model may provide an additional tool in planning and evaluation of studies, although its validity will need further research. The systematic review also identified a paucity of published quality improvement interventions in this regard and the quality improvement model may be able to develop better educational learning outside the constraints which are likely to hamper prospective RCTs. The 'seeone, do-one' method has its limitations and the use of simulation, ultrasound, and standardising teaching have been shown to have significant positive outcomes as educational interventions. 
The evidence highlights that low cost, low-fidelity technology can be just as effective, and should be considered carefully in resource and budget planning for departments and universities. Ultrasound should be considered as a first-line teaching tool for novice trainees and considering the ease of access to point-ofcare ultrasound which is currently available within theatre suites, it could be easily achieved. Learning curves remain an important piece of knowledge for trainers to understand to allow for effective coaching of a trainee. Mental imagery may help trainees rehearse a procedure in advance, and trainers should ensure that any general feedback that is delivered is post-procedure to allow maximum absorption and effect, however the authors recognise that there may however be times where a patient is at risk of harm where the trainer needs to intervene with real-time feedback. Planning and reflection may influence the quality and outcomes, of both learning and research quality. The adapted Kirkpatrick reflective model proposed in this research will require validation, which is beyond the scope of this paper.

\section{Ethics}

An application to the Research Ethics Committee (REC) at the University of Exeter Medical School (UEMS) was undertaken and approved in February 2018.

\section{Acknowledgements}

Professor Karen Mattick, Master of Science in Clinical Education (MClinEd) Lead, University of Exeter Medical School, Exeter, United Kingdom-for their help, advice, guidance, and support with manuscript preparation for submission for publication. Ms. Sophie Robinson, Information Specialist, University of Exeter, UKfor their help and guidance with search strategy.

\section{Declaration}

This paper was presented in part, in poster format at the Association for the Study of Medical Education (ASME) International Conference, Glasgow, 3-5 $5^{\text {th }}$ July 2019.

\section{References}

1. Reed DA, Beckman TJ, Wright SM (2009) An assessment of the methodologic quality of medical education research studies published in The American Journal of Surgery. Am J Surg (3): 442-444.

2. Gray AT (2018) Atlas of Ultrasound-Guided Regional Anaesthesia E-Book.

3. Ballantyne JC, Carr DB, deFerranti S, Suarez T, Lau J, et al. (1998) The comparative effects of postoperative analgesic therapies on pulmonary outcome: cumulative meta-analyses of randomized, controlled trials. Anesth Analg 86(3): 598-612.

4. Rodgers A, Walker N, Schug S, McKee A, Kehlet H, et al. (2000) Reduction of postoperative mortality and morbidity with epidural or spinal anaesthesia: results from overview of randomised trials. BMJ 321(7275): 1493.

5. Cook T, Counsell D, Wildsmith J (2009) Major complications of central neuraxial block: report on the Third National Audit Project of the Royal College of Anaesthetists. Br J Anaesth 102(2): 179-190.

6. Gleeson C, Reynolds F (1998) Accidental dural puncture rates in UK obstetric practice. Int J Obstet Anesth 7(4): 242-246.
7. (2010) Anaesthetists RCo. Curriculum for a CCT in Anaesthetics.

8. Irvine D (2006) A short history of the General Medical Council. Med Educ 40(3): 202-211.

9. Dang T, Annaswamy TM, Srinivasan MA (2001) Development and evaluation of an epidural injection simulator with force feedback for medical training. Stud Health Technol Inform 81: 97-102.

10. Grantcharov TP, Reznick RK (2008) Teaching rounds: teaching procedural skills. BMJ 336(7653): 1129.

11. Kopta JA (1971) The development of motor skills in orthopaedic education. Clin Orthop Relat Res 75: 80-85.

12. Hutton I, Kenealy H, Wong C (2008) Using simulation models to teach junior doctors how to insert chest tubes: a brief and effective teaching module. Intern Med J 38(12): 887-891.

13. McLeod PJ, Steinert Y, Trudel J, Gottesman R (2001) Seven principles for teaching procedural and technical skills. Acad Med 76(10): 1080.

14. Bould M, Crabtree N, Naik V (2009) Assessment of procedural skills in anaesthesia. Br J Anaesth 103(4): 472-483.

15. Lorello GR, Cook DA, Johnson RL, Brydges R (2014) Simulation-based training in anaesthesiology: a systematic review and meta-analysis. $\mathrm{Br}$ J Anaesth 112(2): 231-245.

16. Moher D, Liberati A, Tetzlaff J, Altman DG (2009) Preferred reporting items for systematic reviews and meta-analyses: The prisma statement. PLoS Med 151(4): 264-269.

17. Urwin S, Parker M, Griffiths R (2000) General versus regional anaesthesia for hip fracture surgery: a meta-analysis of randomized trials. Br J Anaesth 84(4): 450-455.

18. Sites BD, Chan VW, Neal JM, Weller R, Grau T, et al. (2010) The American Society of Regional Anaesthesia and Pain Medicine and the European Society of Regional Anaesthesia and Pain Therapy Joint Committee Recommendations for Education and Training in Ultrasound-Guided Regional Anaesthesia. Reg Anesth Pain Med 35(2): 40-46.

19. Reed DA, Cook DA, Beckman TJ, Levine RB, Kern DE, et al. (2007) Association between funding and quality of published medical education research. JAMA 298(9): 1002-1009.

20. Steinert Y, Mann K, Centeno A, Dolmans D, Spencer J, et al. (2006) A systematic review of faculty development initiatives designed to improve teaching effectiveness in medical education: BEME Guide No. 8. Med Teach 28(6): 497-526.

21. Higgins JPT, Altman DG, Gøtzsche PC, Jüni P, Moher D, et al. The Cochrane Collaboration's tool for assessing risk of bias in randomised trials. BMJ 343: 5928.

22. Rothstein HR, Sutton AJ, Borenstein M (2006) Publication bias in metaanalysis: Prevention, assessment and adjustments. John Wiley \& Sons.

23. Naik VN, Devito I, Halpern SH (2003) Cusum analysis is a useful tool to assess resident proficiency at insertion of labour epidurals. Can J Anaesth 50(7): 694-698.

24. Kulcsár Z, O'Mahony E, Lövquist E, Annette Aboulafia , Daša Šabova et al. (2013) Preliminary evaluation of a virtual reality-based simulator for learning spinal anaesthesia. Journal of clinical anaesthesia. 25(2): 98-105.

25. Grau T, Bartusseck E, Conradi R, Martin E, Motsch J (2003) Ultrasound imaging improves learning curves in obstetric epidural anaesthesia: a preliminary study. Can J Anaesth 50(10): 1047-1050.

26. Friedman Z, Siddiqui N, Katznelson R, Devito I, Bould et al. (2009) Clinical impact of epidural anaesthesia simulation on short- and longterm learning curve: High- versus low-fidelity model training. Reg Anesth Pain Med 34(3): 229-232. 
27. Friedman Z, Siddiqui N, Mahmoud S, Davies S (2013) Video-assisted structured teaching to improve aseptic technique during neuraxial block. Br J Anaesth 111(3): 483-487.

28. Gonzalez-Cota A, Chiravuri S, Stansfield RB, Brummett CM, Hamstra SJ (2013) The effect of bench model fidelity on fluoroscopy-guided transforaminal epidural injection training: a randomized control study. Reg Anesth Pain Med 38(2): 155-160.

29. Deacon AJ, Melhuishi NS, Terblanche NC (2014) CUSUM method for construction of trainee spinal ultrasound learning curves following standardised teaching. Anaesth Intensive Care 42(4): 480-486.

30. VanderWielen BA, Harris R, Galgon RE, VanderWielen LM, Schroeder KM (2015) Teaching sonoanatomy to anaesthesia faculty and residents: utility of hands-on gel phantom and instructional video training models. J Clin Anesth 27(3): 188-194.

31. Lim G, Krohner RG, Metro DG, Rosario BL, Jeong JH, et al. (2016) LowFidelity Haptic Simulation Versus Mental Imagery Training for Epidural Anaesthesia Technical Achievement in Novice Anaesthesiology Residents: A Randomized Comparative Study. Anesth Analg 122(5): 1516-1523.

32. Lean LL, Hong RYS, Ti LK (2017) End-task versus in-task feedback to increase procedural learning retention during spinal anaesthesia training of novices. Adv Health Sci Educ Theory Pract 22(3): 713-721.

33. Akhtar S, Hwang U, Dickman E, Nelson BP, Morrison RS, et al. (2013) A brief educational intervention is effective in teaching the femoral nerve block procedure to first-year emergency medicine residents. J Emerg Med 45(5): 726-730.

34. Baranauskas MB, Margarido CB, Panossian C, Silva ED, Campanella MA et al. (2008) Simulation of ultrasound-guided peripheral nerve block: learning curve of CET-SMA/HSL Anaesthesiology residents. Rev Bras Anestesiol 58(2): 106-111.

35. Chen H, Kim R, Perret D, Hata J, Rinehart J, et al. (2016) Improving Trainee Competency and Comfort Level with Needle Driving Using Simulation Training. Pain Medicine 17(4): 670-674.

36. Lim MW, Burt G, Rutter SV (2005) Use of three-dimensional animation for regional anaesthesia teaching: application to interscalene brachial plexus blockade. Br J Anaesth 94(3): 372-377.

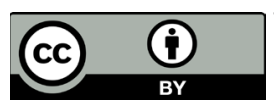

This work is licensed under Creative Commons Attribution 4.0 License

DOI: 10.19080/JAICM.2020.10.555791
37. Genesis. Epidural-Spinal Injection Simulator.

38. Greatbatch D (2018) A false economy.

39. Paech MJ, Godkin R, Webster S (1998) Complications of obstetric epidural analgesia and anaesthesia: a prospective analysis of 10995 cases. Int J Obstet Anesth 7(1): 5-11.

40. Reason J (2004) Beyond the organisational accident: the need for "error wisdom" on the frontline. BMJ Quality and Safety 13(2): 28-33.

41. Fitts PM, Posner MI (1967) Learning and skilled performance in human performance.

42. Lave J, Wenger E, Wenger E (1991) Situated learning: Legitimate peripheral participation.

43. Wenger E (1999) Communities of practice: Learning, meaning, and identity.

44. Kirkpatrick DL (1975) Evaluating training programs.

45. Yardley S, Dornan T (2012) Kirkpatrick's levels and education 'evidence'. Med Educ 46(1): 97-106.

46. Shao L, Duan Y, Sun X, Gao H, Zhu D, Miao W Answering who/when, what, how, why through constructing data graph, information graph, knowledge graph and wisdom graph.

47. Wu AW, Lipshutz AK, Pronovost PJ (2008) Effectiveness and efficiency of root cause analysis in medicine. JAMA 299(6): 685-687.

48. Easterbrook PJ, Gopalan R, Berlin J, Matthews DR (1991) Publication bias in clinical research. The Lancet 337 (8746): 867-872.

49. Cook DA, Reed DA (2015) Appraising the quality of medical education research methods: the medical education research study quality instrument and the Newcastle-Ottawa scale-education. Acad Med 90(8): 1067-1076.

50. Littlewood S, Ypinazar V, Margolis SA, Scherpbier A, Spencer J, et al. (2005) Early practical experience and the social responsiveness of clinical education: systematic review. BMJ 331(7513): 387-391.

\section{Your next submission with Juniper Publishers will reach you the below assets}

- Quality Editorial service

- Swift Peer Review

- Reprints availability

- E-prints Service

- Manuscript Podcast for convenient understanding

- Global attainment for your research

- Manuscript accessibility in different formats

( Pdf, E-pub, Full Text, Audio)

- Unceasing customer service

Track the below URL for one-step submission https://juniperpublishers.com/online-submission.php 